\title{
PROGRESS REPORT \\ TO THE U.S. DEPARTMENT OF ENERGY \\ GRANT DE-FG02-91ER40626 \\ Neutrino Physics, Particle Theory and Cosmology
}

Period Covered by this Report: 1 August 2008 - 31 July 2009

Anniversary Date of Grant: 1 February 2009

Date of this Report: 31 July 2009

\section{Overview}

Research conducted under this grant over the past year has been driven by the impending operation of the Large Hadron Collider (LHC), and by the ongoing developments in neutrino physics and cosmology. The recent launch of the Planck satellite should have far reaching implications for cosmology in the coming years. Research topics include particle astrophysics, neutrino physics, grand unified theories, Higgs and sparticle spectroscopy, dark energy and dark matter, inflationary cosmology, and baryo/lepto-genesis. Faculty members on the grant are Stephen Barr, Thomas Gaisser, Qaisar Shafi and Todor Stanev. Ilia Gogoladze and Hasan Yuksel are the two postdoctoral scientists supported by the DOE grant. There are currently several excellent students in our research program. One of them, Mansoor Rehman, has been awarded a competitive university fellowship on which he will be supported from September 1, 2009 - June 30, 2010. Another student, Joshua Wickman, has been awarded a fellowship by the Delaware Space Grant Consortium (in affiliation with NASA), and will be supported by this fellowship from September 1, 2009 - August 31, 2010. Both of these students also attended the TASI Summer School in June 2009, at which they each presented a student talk on topics in inflationary cosmology.

\section{Phenomenology and Model Building}

(Barr, Shafi, postdoc Gogoladze, and graduate students)

Barr and Almas Khan proposed a realistic and highly predictive $S O(10)$ model with a "radiative hierarchy" of quark and lepton masses [1]. Such radiative hierarchy models are difficult to construct in supersymmetric models, which is why little work has been done on them. But the paper of Barr and Khan shows that very simple radiative hierarchy models can be constructed in the context of non-supersymmetric grand unification. All the masses and mixing of second and third family come from just three tree-level Yukawa operators. The first family masses and mixing comes from loop diagrams. Because the model is radiative, the loop factors of $1 / 16 \pi^{2}$ are able to explain the very large range of fermion masses and mixings without the ad hoc introduction of small parameters - all the parameters that come into the fermion mass matrices are of order one. In this paper a full description and analysis of the model is done, including patterns of gaugesymmetry breaking, calculation of the loops that give radiative fermion masses, renormalization group running, calculation of the CKM and MNS angles, and numerical fits to the data. A good chi-squared fit is found. The paper was published in Physical Review D.

Two important parameters of the Standard Model that are still not known are the neutrino mixing angle $\theta_{13}$ and the Dirac CP-violating phase $\delta_{\text {lep }}$ of the leptons. A great deal of experimental effort is going to be spent in the attempt to measure them. In a paper recently submitted to Physics Letters, Barr and Khan show that a definite testable relation can be obtained between these two important angles in a wide class of models that have certain basic features, including $S O(10)$ symmetry and a "lopsided" structure of the mass matrices [2]. The point is that $S O(10)$ tends to relate the Dirac mass matrix of the neutrinos, $M_{\text {Dirac }}$, to the up-quark mass matrix. 
The smallness of the $u$ quark mass then tends to imply that $M_{\text {Dirac }}$ is approximately rank-2. Consequently, the neutrino mass matrix $M_{\nu}=-M_{\text {Dirac }} M_{R}^{-1} M_{\text {Dirac }}^{-1}$ is also nearly rank-2. This, finally, means that the matrix that diagonalizes $M_{\nu}$ it is approximately a $2 \times 2$ unitary matrix, and therefore contains only two unknown physical parameters. In other words, even though the mass matrix of the right-handed neutrinos, $M_{R}$, has many unknown parameters, in effect there are only two that matter in this kind of model. That is why a testable relation between $\theta_{13}$ and $\delta_{l e p}$ results. Barr and Khan illustrate these ideas in the context of a specific non-supersymmetric $S O(10)$ model, and graph the relation between $\theta_{13}$ and $\delta_{l e p}$ by doing a detailed numerical fit.

In a recently completed paper, we (I. Gogoladze, M. U. Rehman and Q. Shafi) studied the little hierarchy problem that is encountered in the constrained minimal supersymmetric model (CMSSM), and showed that this problem can be ameliorated in models based on the gauge symmetry $G_{422} \equiv \mathrm{SU}(4)_{c} \times \mathrm{SU}(2)_{L} \times \mathrm{SU}(2)_{R}[3]$. The standard assumption in the CMSSM (as well as in $\mathrm{SU}(5)$ and $\mathrm{SO}(10)$ ) of universal gaugino masses can be relaxed in $G_{422}$. Doing so results in a significant improvement in the degree of fine tuning required to implement radiative electroweak breaking in the presence of a characteristic supersymmetry breaking scale of around a TeV. The paper presents examples of Higgs and sparticle mass spectra that are realized with $10 \%$ fine tuning, a significant improvement over the $0.1 \%$ or so fine tuning required in the CMSSM.

In the last year, we (K.S. Babu, Ilia Gogoladze, Mansoor Ur Rehman and Qaisar Shafi) have investigated the impact of $\mathrm{TeV}$-scale matter belonging to complete vectorlike multiplets of unified groups on the lightest Higgs boson in the MSSM [4]. This study showed that the mass $m_{h}$ of the lightest Higgs can be as large as $160 \mathrm{GeV}$, which is consistent with perturbative unification and electroweak precision data. For lower values $m_{h}<125 \mathrm{GeV}$, these extended MSSM models can also render the little hierarchy problem less severe. As part of this investigation, the sparticle mass spectrum in these models was also estimated.

Shafi and Gogoladze, working with collaborator Nobuchika Okada and graduate student Rizwan Khalid, have explored the sparticle and Higgs spectroscopy of an SU(5) inspired extension of the constrained minimal supersymmetric standard model (CMSSM) [5]. This extension involves replacing universal soft parameter $m_{0}$ by $m_{\overline{5}}$ and $m_{10}$, where $m_{\overline{5}}$ and $m_{10}$ denote universal soft scalar masses associated with fields in the five and ten dimensional representations of SU(5). They have found that the special case $m_{\overline{5}} \ll m_{10}$ yields a rather characteristic sparticle spectroscopy which can be tested at the LHC. The paper highlights a few benchmark points in which the lightest neutralino saturates the WMAP bound on cold dark matter abundance.

In another paper with Okada, Shafi and Gogoladze have considered extensions of the next-tominimal supersymmetric model (NMSSM) in which the observed neutrino masses are described in terms of effective dimension six (or seven) rather than dimension five operators [6]. All such operators respect the discrete symmetries of the model. The new particles associated with the double (or triple) seesaw mechanism can have sizable couplings to the known leptons, even with a $\mathrm{TeV}$ seesaw scale. In the latter case some of these new short-lived particles could be produced and detected at the LHC.

\subsection{Publications (August 1, 2008 - July 31, 2009)}

1. A Realistic Radiative Fermion Mass Hierarchy in Non-supersymmetric $\mathrm{SO}(10)$ S.M. Barr and Almas Khan Phys. Rev. D 79, 115005 (2009), [arXiv:0807.5112 [hep-ph]].

2. A Relation between $\theta_{13}$ and the Leptonic Dirac CP Phase in SO(10) Lopsided Models S.M. Barr and Almas Khan arXiv:0906.0535 [hep-ph].

3. Amelioration of Little Hierarchy Problem in $S U(4)_{c} \times S U(2)_{L} \times S U(2)_{R}$ I. Gogoladze, M. Ur Rehman, Q. Shafi arXiv:0907.0728 [hep-ph]. 
4. Higgs Boson Mass, Sparticle Spectrum and Little Hierarchy Problem in Extended MSSM K.S. Babu, I. Gogoladze, M. ur Rehman, Q. Shafi

Phys. Rev. D 78, 055017 (2008).

5. Soft Probes of SU(5) Unification

I. Gogoladze, R. Khalid, N. Okada and Q. Shafi

Phys. Rev. D 79, 095022 (2009), [arXiv:0811.1187 [hep-ph]].

6. NMSSM and Seesaw Physics at LHC

I. Gogoladze, N. Okada and Q. Shafi

Phys. Lett. B 672, 235 (2009), [arXiv:0809.0703 [hep-ph]].

7. Doubly Lopsided Mass Matrices from Unitary Unification

S.M. Barr

Phys. Rev. D 78, 075001 (2008).

\subsection{Work in Progress}

- Relation between $\theta_{13}$ and $\delta_{l e p}$

Barr and Khan are extending their work on the relation between $\theta_{13}$ and $\delta_{l e p}$ in a class of "lopsided" $S O(10)$ models by doing a detailed numerical fit to a supersymmetric $S O(10)$ model proposed by Babu and Barr in 2002 that happens to belong in this class. Though Babu and Barr were unaware of this in 2002, this model should have a distinctive and testable relation between these two angles.

\section{- Minimal $S O(10)$ and Exogenous Mixing}

Barr and Dorsner are collaborating on a project involving a new idea for understanding quark and lepton masses that allows the construction of highly testable models. The idea is to combine two older ideas: (a) so-called "minimal $S O(10)$ ", which has been extensively studied in recent years, and (b) "exogenous mixing", which was proposed in 1995 by Babu and Barr. In exogenous mixing, the "underlying mass matrices" of the "unmixed" quarks and leptons have no interfamily hierarchy: all their non-vanishing elements are of the same order. However, these quarks and leptons mix with other vectorlike fermions, and the mixing causes these underlying mass matrices to get multiplied by matrices that are hierarchical.

\section{- Testing SU(5) and Flipped SU(5) Unification at LHC}

This work is being carried out by two graduate students Shabbar Rizvi and Rizwan Khalid under the supervision of Shafi and Gogoladze. The main idea here is to exploit the soft supersymmetry breaking sector as a probe of the the underlying unified gauge symmetry groups. For instance, while in $\mathrm{SO}(10)$ the scalar particles in the 16 dimensional representation all acquire a universal soft mass terms, the 10 and $\overline{5}$ multiplets can have distinct soft scalar masses. This can be tested at the LHC by finding both squarks and sleptons, in the same manner in which grand unification is being tested by measuring the SM gauge couplings at low energies. We plan to make comparisons between $\mathrm{SO}(10), \mathrm{SU}(5)$ and flipped $\mathrm{SU}(5)$ models.

\section{- Electroweak Baryogenesis and Light Stop}

We plan to explore supersymmetric models of electroweak baryogenesis which naturally contain a light stop quark. This is challenging for the MSSM which requires heavy stops in order to obtain a Higgs mass which is compatible with the LEP2 bound of $114.4 \mathrm{GeV}$. To realize this program we will explore extensions of the MSSM which contain new vectorlike multiplets. These models retain gauge coupling unification, allow for a suitably massive Higgs and may permit one of the stop quarks to be in the range required by electroweak baryogenesis. The new vectorlike particles play an essential role in realizing this program. These models also help in ameliorating the little hierarchy problem. 


\title{
3 Cosmology and Particle Astrophysics Theory
}

\author{
(Barr, Gaisser, Shafi, Stanev, postdocs Gogoladze and Yuksel, and graduate students)
}

The work of T.K. Gaisser and T. Stanev concentrated on two main issues: extending the spectrum of atmospheric neutrinos, and studies of the extensions of hadronic interaction models to ultrahigh energy. The extension of the atmospheric neutrino production went in two directions. Gaisser teamed up with colleagues in the University of Wisconsin to estimate the fluxes of prompt neutrinos from decays and charm and higher flavors. The work resulted in a code that predicts the atmospheric neutrino fluxes to energies as high as $1000 \mathrm{TeV}$. Gaisser considered properties of atmospheric neutrinos in the $10 \mathrm{TeV}$ range in connection with proposals to use such neutrinos as a probe of the core-mantle transition of the Earth [1].

On the other hand Gaisser and Stanev work with G. Barr (Oxford University) on calculations of the atmospheric neutrino flux down to $1 \mathrm{MeV}$. This is an important background to the neutrinos from cosmological supernova explosions which the neutrino telescopes are trying to estimate.

The work on the extension of the hadronic interaction models to ultrahigh energies started with a study of the quality of the widely used in air shower analysis interaction model SIBYLL 2.1 [2]. The results of these studies was written up and submitted to Phys. Rev. D. It describes the achivements, as well as the problems the model has in description of the highest energy interactions and the development of extensive air showers at very high energy. Part of the work was the inclusion of charm production in SIBYLL 2.1, which is now available.

Shafi and graduate students Mansoor ur Rehman and Joshua Wickman have published a paper in which they employed Coleman-Weinberg and Higgs potentials to implement inflation in nonsupersymmetric grand unified theories (GUTs) such as $\mathrm{SU}(5)$ and $\mathrm{SO}(10)$ [3]. To realize a scalar spectral index close to 0.96 (as indicated by the most recent WMAP 5-year anlaysis), the energy scale of observable inflation turns out to be of order $10^{16} \mathrm{GeV}$. This implies a GUT symmetry breaking scale of similar magnitude, and proton lifetime of order $10^{34}-10^{38}$ years. In some $\mathrm{SO}(10)$ models with axion dark matter, the scalar leptoquark boson exchange leads to proton decay with a lifetime of order $10^{34}-10^{35}$ years. These predictions of the proton lifetime are somewhat above the current experimental bound set by Super-K, and can be probed by the next generation of detectors.

Also in the past year, Shafi, Rehman and Wickman have published a paper in which they studied the effects of including one-loop radiative corrections in a non-supersymmetric hybrid inflationary model [4]. These corrections can arise from Yukawa couplings between the inflaton and right-handed neutrinos, and induce a maximum in the potential which admits hilltop-type solutions in addition to the standard hybrid solutions. It is possible to obtain a red-tilted spectral index $n_{s}$, consistent with WMAP5 data, for sub-Planckian values of the field. This is in contrast to the tree level analysis, in which a red-tilted spectrum is achieved only for trans-Planckian values of the field. This outcome is similar to the case of chaotic inflation, examined by Shafi and former graduate student Nefer Senoguz [5], in which the one-loop model exhibited a reduced tensor-to-scalar ratio $r$ relative to the tree level case.

Gogoladze and Shafi, in collaboration with Okada, recently proposed a simple extension of the Standard Model in which type II seesaw physics enables the dark matter particle (WIMP) to acquire leptophilic properties [6]. The implications of this scheme for the ongoing PAMELA, ATIC and Fermi observations are currently being investigated.

In reference [7], written in collaboration with graduate student Rizwan Khalid, it is shown that third family Yukawa unification and the existence of neutralino dark matter are perfectly compatible with each other. This is in sharp contrast to the $\mathrm{SO}(10)$ model in which such a scenario is difficult, if not impossible, to realize.

I. Gogoladze, R. Khalid, Q. Shafi and H. Yuksel showed that dark matter neutralinos in the constrained minimal supersymmetric model (CMSSM) may also account for the recent cosmic ray electron and positron observations reported by the PAMELA and other experiments either through self annihilation or via decay [8]. However, to achieve this, both scenarios require new physics beyond the 'standard' CMSSM, and a unified explanation suggests a neutralino mass of 
order $700 \mathrm{GeV}-2 \mathrm{TeV}$. We study the implications of these scenarios for Higgs and sparticle spectroscopy in the CMSSM and highlight some benchmark points.

The surprising excesses of positrons and electrons recently detected by the PAMELA and Fermi space experiments suggest that a source of such cosmic rays must reside within our neighborhood of the Milky Way. H. Yuksel and T. Stanev with M. D. Kistler from the Ohio State University showed that the unexpected "halo" of very-high-energy gamma rays recently observed to surround the Geminga pulsar (a rapidly-rotating neutron star) may be essential to resolving this puzzle [9]. We conclude that these gamma rays imply that a wind of highly-energetic cosmic rays is escaping from Geminga, confirming the presence of a powerful particle accelerator near the Earth. Cosmic rays produced in Geminga's active past are, after a circuitous journey through the Galaxy's tangled magnetic fields, likely the mysterious "Positron Excess." Stanev also participated in another study in which cosmic ray electron and cosmic ray positron excesses are attributed to the acceleration in the supernova shock in the polar cap of exploding Wolf Rayet and Red Super Giant stars [10]. Both studies are accepted by Phys. Rev. Letters.

High-redshift gamma-ray bursts (GRBs) offer an extraordinary opportunity to study aspects of the early Universe, including the cosmic star formation rate (SFR). Motivated by the two recent highest- $z$ GRBs, GRB 080913 at $z=6.7$ and GRB 090423 at $z=8.1$, and more than four years of Swift observations, H. Yuksel (together with M. D. Kistler and J. F. Beacom from the Ohio State University, and A. M. Hopkins of the Anglo-Australian Observatory and J. S. Wyithe of University of Melbourne) first confirm that the GRB rate does not trace the SFR in an unbiased way [11]. Correcting for this, we find that the implied SFR to beyond $z=8$ is consistent with LBG-based measurements after accounting for unseen galaxies at the faint end of the UV luminosity function. We show that this provides support for the integrated star formation in the range $6<z<8$ to have been alone sufficient to reionize the Universe.

\subsection{Publications (August 1, 2008 - July 31, 2009)}

1. Atmospheric neutrinos in the context of muon and neutrino radiography Thomas K. Gaisser Accepted for publication in Earth, Planets, Space vol 61 [arXiv:0901.2386 [astro-ph]].

2. Hadronic Interaction Model Sibyll 2.1 E.-J. Ahn, R. Engel, T.K. Gaisser, P. Lipari, T. Stanev Submitted to Phys. Rev. D, [arXiv:0906.4113 [hep-ph]].

3. GUT Inflation and Proton Decay after WMAP5 M. Ur Rehman, Q. Shafi and J. R. Wickman Phys. Rev. D 78, 123516 (2008), [arXiv:0810.3625 [hep-ph]].

4. Hybrid Inflation Revisited in Light of WMAP5 M. Ur Rehman, Q. Shafi and J. R. Wickman Phys. Rev. D 79, 103503 (2009), [arXiv:0901.4345 [hep-ph]].

5. Chaotic inflation, radiative corrections and precision cosmology V. N. Senoguz and Q. Shafi Phys. Lett. B 668, 6 (2008).

6. Type II Seesaw and the PAMELA/ATIC Signals I. Gogoladze, N. Okada, Q. Shafi arXiv:0904.2201 [hep-ph].

7. Yukawa Unification and Neutralino Dark Matter in $S U(4)_{c} \times S U(2)_{L} \times S U(2)_{R}$ I. Gogoladze, R. Khalid, Q. Shafi Phys. Rev. D 79, 115004 (2009), [arXiv:0903.5204 [hep-ph]]. 
8. CMSSM Spectroscopy in light of PAMELA and ATIC

I. Gogoladze, R. Khalid, Q. Shafi and H. Yuksel

Phys. Rev. D 79, 055019 (2009), [arXiv:0901.0923 [hep-ph]].

9. TeV Gamma Rays from Geminga and the Origin of the GeV Positron Excess

H. Yuksel, M.D. Kistler and T.Stanev

Phys. Rev. Lett. accepted, [arXiv:0810.2784 [astro-ph]].

10. Cosmic ray positrons and electrons

P.L. Biermann, J.K. Becker, A. Meli, W. Rhode, E.-S. Seo, T. Stanev

Phys. Rev. Lett. accepted, [arXiv:0903.4048 [astro-ph.HE]].

11. The Star Formation Rate in the Reionization Era as Indicated by Gamma-ray Bursts

M. D. Kistler, H. Yuksel, J. F. Beacom, A. M. Hopkins and J. S. B. Wyithe

arXiv:0906.0590 [astro-ph.CO].

12. Cosmic Rays

T.K. Gaisser and T. Stanev

Review of Particle Physics (C. Amsler et al.), Phys. Lett. B 667, 1 (2008).

13. Core-Collapse Astrophysics with a Five-Megaton Neutrino Detector

M. D. Kistler, H. Yuksel, S. Ando, J. F. Beacom and Y. Suzuki

arXiv:0810.1959 [astro-ph].

14. Propagation of Ultrahigh Energy Cosmic Rays

T. Stanev

New J. Phys. 11, 065013 (2009), [arXiv:0810.2501 [astro-ph]].

\subsection{Work in Progress}

\section{- Dark Matter from Rank > 4 Grand unification}

Babu, Barr, and Gogoladze are working on a paper showing that dark matter candidates naturally arise from grand unification, if the unification group has rank greater than 4, i.e. for any unification group other than $S U(5)$. The point is that for all such groups there are fermions in the same GUT multiplets with the known quarks and leptons that are singlets under the Standard Model group. There are also extra $U(1)$ gauge symmetries contained in the unified group that can keep these extra singlet fermions light (i.e. near the $\mathrm{TeV}$ scale). Moreover, the "extra $Z$ boson(s)" of such extra $U(1)$ 's can be the means by which the light singlet fermions annihilate, leaving a relic abundance consistent with them being the dark matter. An interesting relation between the mass of the extra $Z$ boson and the mass of the dark matter particle can be computed for any given model of this type.

\section{- Improving SIBYLL in the LHC era}

Future work, to be conducted during the next year by Gaisser and Stanev, will include corrections of all problems that were identified in the current version (2.1) of the SIBYLL code. Predictions of the improved SIBYLL 3.XX models will be compared with the results of the LHC, where most of the attention will be on the forward detectors TOTEM and LHCf that will be active in the very beginning of the LHC runs.

\section{- Energy \& Zenith Angle Dependence of the Atmospheric Neutrino Fluxes}

The dependence of the atmospheric neutrino fluxes on the zenith angle and energy can be utilized to distinguish signals of diffuse extraterrestrial flux of high-energy neutrinos that could arise from WIMP annihilation in the Sun or other cosmological sources. This problem is currently being investigated by T. K. Gaisser and H. Yuksel. 


\section{- Yukawa Unification and Neutralino Dark Matter}

Shafi, Gogoladze and graduate student Rizwan Khalid are exploring the low energy implications of imposing third family Yukawa unification. More specifically, they are investigating $\mathrm{SO}(10)$ and $\mathrm{E}_{6}$ based supersymmetric models as well as several subgroups such as $\mathrm{SU}(4)_{c} \times \mathrm{SU}(2)_{L} \times \mathrm{SU}(2)_{R}$ and $\mathrm{SU}(3)_{c} \times \mathrm{SU}(3)_{L} \times \mathrm{SU}(3)_{R}$. One of the main goals is to explore the TeV scale Higgs and sparticle spectroscopy of these models which can be tested at the LHC.

\section{- Supersymmetry and GeV-Scale Dark Matter}

The DAMA experiment has reported events which could be interpreted as signaling the existence of a $7 \mathrm{GeV}$ neutralino dark matter. It should be noted that there is no independent verification of the DAMA events. The assumption of universal gaugino masses in the CMSSM means that the DAMA events cannot be explained in this scheme. However, there are many realistic supersymmetric models in which the assumption of universal gaugino masses is too restrictive. These include models based on flipped $\mathrm{SU}(5)$, and so our goal is to explore the parameter space offered in such models. This project will be carried out in collaboration with graduate student Shabbar Rizvi. The wider consequences for Higgs and sparticle spectroscopy will also be explored. Besides a light neutralino we also expect to find relatively light sparticles which could be discovered at the LHC.

\section{- Supersymmetric Dark Matter, Theory versus Observations}

The existence of dark matter is certain, but the properties of the dark matter particles are only poorly constrained, with several attractive but rather different candidates. Q. Shafi, I. Gogoladze, H. Yuksel together with graduate students Khalid and Rizvi will cover a wide variety of realistic particle physics models by allowing for different scenarios of supersymmetry breaking and distinct attractive gauge groups such as $\mathrm{SO}(10)$ and $\mathrm{SU}(5)$. We expect them to yield a variety of signatures which can be tested and distinguished with ongoing and upcoming experiments. We are also pursuing less conventional particle physics dark matter models in order to understand the recent results reported by PAMELA and ATIC. This could allow for a fairly complete characterization of particle dark matter candidates in terms of their mass, annihilation cross section and lifetimes, which is necessary to bridge the gap between particle physics and astrophysical understanding of this challenge.

\section{- Inflation Models, Radiative Corrections and Precision Cosmology}

Graduate students Mansoor Rehman and Joshua Wickman, together with Shafi, are studying a variety of realistic inflationary models, taking into account the presence of (additional) couplings which are required to reheat the universe at the end of inflation. Through radiative corrections, these couplings can provide important modifications of the inflaton potential. As a consequence, the naive tree level predictions can be significantly altered. The PLANCK satellite is expected to provide stringent tests of such models in the near future.

\section{- Axion Inflation in Non-Minimal Models}

Shafi, Okada (KEK, Japan) and graduate student Rehman are investigating the possibility of implementing axion driven inflation with non-minimal couplings. An approach such as this was recently attempted by several authors in the framework of the Standard Model with the Higgs boson playing the role of the inflaton. Alas, this SM based scenario does not quite work. The problem is that in the presence of a large dimensionless parameter $\left(\sim 10^{4}\right.$ in order to realize inflation), the required energy scale for inflation turns out to be significantly greater (by a factor $\sim 10^{2}$ ) than the effective cutoff scale. We are optimistic that this problem can be circumvented in axion based inflation.

\section{- Anisotropies of the Ultra High Energy Cosmic Rays}

The Pierre Auger Southern Observatory has shown that the all-sky distribution of the highest energy cosmic rays might not be isotropic, but instead correlated with known astrophysical sources such as Active Galactic Nuclei within $100 \mathrm{Mpc}$. This apparent correlation might 
provide clues about the origin of the ultra high energy cosmic rays, their acceleration mechanism and the conditions in their sources. T. Stanev and H. Yuksel are currently working on this project.

\section{Invited/Contributed Talks (August 1, 2008 - July 31, 2009)}

1. Prediction of $\theta_{13}$ and Leptonic CP Violation in a Class of $S O(10)$ Models

S.M. Barr and Almas Khan

SUSY 2009, Boston (June 7, 2009).

2. Flavor Structure from $S U(N)$ Unification

S.M. Barr

Vanderbilt University (March 18, 2009).

3. Horizontal (Flavor) Symmetry from Vertical (Gauge) Symmetry

S.M. Barr

Miami 2008, Fort Lauderdale, Florida (December 17, 2008).

4. Two lectures on Cosmic Rays in Large Air Shower Detectors presented at the Summer School on Particle and Nuclear Astrophysics

Thomas K. Gaisser

Institute for Nuclear Theory, Seattle, WA (July 1 and 2, 2009).

5. Atmospheric Muons and Neutrinos in Neutrino Telescopes: the Role of Hadronic Interactions at High Energy

Thomas K. Gaisser

Tenth Workshop on Non-Perturbative QCD, Paris, France (June 9, 2009).

6. Yukawa Unification and Neutralino Dark Matter in Pati-Salam Model

Ilia Gogoladze

Pheno 2009 symposium, Madison, Wisconsin (May 12, 2009).

7. Soft Probes of SU(5) Unification

Ilia Gogoladze

Miami 2008, Fort-Lauderdale, Florida (December 19, 2008).

8. Susy Hybrid Inflation in the Light of WMAP5

Mansoor Ur Rehman

TASI 2009, University of Colorado at Boulder (June 12, 2009).

9. Inflationary Cosmology and Particle Physics

Qaisar Shafi

Hengstberger Symposium: Extra Dimensions and Mini Black Holes (July 2009, Heidelberg).

10. Higgs Boson And New Physics At The LHC

Qaisar Shafi

Kavli Institute (June 2009, Beijing).

11. Lectures presented at the 2009 BCVSPIN Summer School In Particle Physics And Cosmology Qaisar Shafi

BCVSPIN 2009 (June 2009, Beijing).

12. Higgs Mass Bounds in the Light of Neutrino Oscillation

Qaisar Shafi

Miami 2008, Fort-Lauderdale, Florida (December 19, 2008). 
13. Higgs Mass Bounds in the Light of Neutrino Oscillation Qaisar Shafi

ICHEP08, University of Pennsylvania, Philadelphia, PA (August 2008).

14. Four lectures presented at the 2009 ICTP summer school

T. Stanev

ICTP Summer school on Particle Physics (June 2009).

15. Ultrahigh Energy Cosmic Rays: Origin and propagation

T. Stanev

XXI Rencontre de Blois, Blois, France (June 2009).

16. High energy cosmic rays and extensive air showers

T. Stanev

Elastic and Diffractive Scattering workshop, CERN, Geneva (June 2009).

17. Recent Progress and new problems in Cosmic ray physics

T. Stanev

April Meeting of APS, Denver, (May 2009).

18. Ultra-high Energy Cosmic Rays and Magnetic Fields in the Universe

T. Stanev

Ultra-high Energy Cosmic Rays: Theory and observations Ringberg Castle, Germany (February 2009).

19. Open problems in cosmic rays and astrophysics;

Measurement methods: muon bundles

T. Stanev

Corsika school 2009, Freudenstadt-Lauterbad, Germany (November 2008).

20. Non-SUSY Inflationary Models and Precision Cosmology

Joshua R. Wickman

TASI 2009, University of Colorado at Boulder (June 12, 2009).

21. The Origin of the Cosmic Positron \& Electron Excesses in Light of the Recent Observations Hasan Yuksel

New Lights on Dark Matters, Perimeter Institute (June 2009).

22. Core-Collapse Astrophysics with Multi Megaton Neutrino Detectors

Hasan Yuksel

Frontiers in Theoretical Neutrino Physics, Paris (March 2009).

23. Core-Collapse Astrophysics with Multi Megaton Neutrino Detectors

Hasan Yuksel

XIII International Workshop on Neutrino Telescopes, Venice (March 2009).

24. Revealing the High-Redshift Star Formation Rate with Gamma-Ray Bursts

Hasan Yuksel

Institute of Nuclear Physics Seminars, Orsay (March 2009).

25. The Origin of the Cosmic Positron \& Electron Excesses in Light of the Recent Observations Hasan Yuksel

HETEP Seminar, University of Illinois at Urbana-Champaign (February 2009).

26. The Origin of the Cosmic Positron \& Electron Excesses in Light of the Recent Observations Hasan Yuksel

High Energy Physics/Astrophysics Seminars, Penn State University (January 2009). 
27. Energy \& Zenith Angle Dependence of Atmospheric Neutrino Fluxes Hasan Yuksel

Novel Searches for Dark Matter, The Ohio State University (November 2008).

28. Core-Collapse Astrophysics with Supernova Neutrinos

Hasan Yuksel

Workshop on Underground Detectors Investigating Grand Unification,

Brookhaven National Laboratory (October 2008).

29. TeV Gamma Rays From Geminga and Multi-GeV Positron Excess

Hasan Yuksel

The impact of high-energy astrophysics experiments on cosmological physics,

KICP Chicago (October 2008).

30. Neutrinos: Candidates and Probes of Dark Matter

Hasan Yuksel

Neutrino Frontiers, University of Minnesota (October 2008).

31. Revealing the High-Redshift Star Formation Rate (and more) with Gamma Ray Bursts

Hasan Yuksel

Cosmo 2008, Madison WI (August 2008).

\section{Conference Proceedings (August 1, 2008 - July 31, 2009)}

1. Doubly Lopsided Models from SUSY SU(N)

S.M. Barr

16th International Conference on Supersymmetry and the Unification of Fundamental Interactions (SUSY08),

Seoul, Korea, 16-21 Jun 2008.

AIP Conf. Proc. 1078:420-424 (2009).

2. RICAP-07: Summary comments

T.K. Gaisser

Nucl. Instrum. Meth. A 588, 276-280 (2008).

3. Higgs boson mass, neutrino oscillations and inflation

Qaisar Shafi

AIP Conf. Proc. 1078 (2009) 49.

4. Higgs boson mass, new physics and inflation

Qaisar Shafi

AIP Conf. Proc. 1015 (2008) 243.

5. Higgs Boson And New Physics At The LHC

Qaisar Shafi

AIP Conf. Proc. 1006 (2008) 20.

6. Active Galactic Nuclei with Starbursts: Sources for Ultra High Energy Cosmic Rays T. Stanev (P. L. Biermann et al.)

Proceedings of "High-Energy Gamma-rays and Neutrinos from Extra-Galactic Sources", Heidelberg, arXiv:0904.1507 [astro-ph.HE].

7. Active Galactic Nuclei: Sources for ultra high energy cosmic rays?

T. Stanev (P. L. Biermann et al.)

Proc. of CRIS 2008 (Malfa, Sicily), Nucl. Phys. B (Proc. Suppl.) 190, 61 (2009), arXiv:0811.1848 [astro-ph]. 
8. The beginning of cosmic ray astronomy

T. Stanev

Concluding remarks at the 2008 Vulcano workshop, "Frontier objects in astrophysics and particle physics", F. Giovannelli \& G. Mannocchi (eds.), Italian Physical Society, Editrice Compositori, Bologna, Italy, arXiv:0808.1236 [astro-ph].

9. Ultra high energy cosmic rays and neutrinos after Auger

T. Stanev

To appear in the Proceedings of the 2008 Vulcano workshop, "Frontier objects in astrophysics and particle physics", F. Giovannelli \& G. Mannocchi (eds.), Italian Physical Society, Editrice Compositori, Bologna, Italy, arXiv:0808.1045 [astro-ph].

10. Core-Collapse Astrophysics with Multi Megaton Neutrino Detectors

Hasan Yuksel

XIII International Workshop on Neutrino Telescopes, Venice (March 2009). 